\title{
Slow-growing and Linearly Spreading Cutaneous Lesion: Often Misdiagnosed Mycobacterium Marinum Infection
}

Lakshmisree A. Vemulakonda $^{1}$, Jamie A. Tschen ${ }^{2}$

1. Pathology, University of Washington, Bothell, USA 2. Dermatopathology, St. Joseph Hospital, Houston, USA

$\square$ Corresponding author: Lakshmisree A. Vemulakonda, vakhila84@gmail.com Disclosures can be found in Additional Information at the end of the article

\section{Abstract}

Mycobacterium marinum is a slow-growing atypical mycobacterium. It is a photochromogen; when exposed to light, it produces yellow pigment. In humans, it manifests as a localized granuloma or sporotrichotic lymphangitis. Patients at risk include anglers (commercial, recreational), oyster workers, swimmers, aquarium workers, and individuals with aquariums in their homes. Herein, we report a case of a Mycobacterium marinum infection which was misdiagnosed because there was no histopathological evidence of acid-fast bacilli and the slow growth rate in cultures.

Categories: Dermatology, Pathology

Keywords: mycobacterium marinum, fish tank granuloma, sporotrichoid spread

\section{Introduction}

Mycobacterium marinum is an atypical mycobacterium that causes dermatological and osteoarticular lesions. The main source of infection is exposure to the aquatic environment or marine life. Despite an increase in the number of cases in recent years, this infection often goes unrecognized or misdiagnosed due to the non-specific clinical and histopathological features and the slow rate of growth in cultures leading to delayed management [1]. In this case report, we would like to familiarize the reader with this infection and its management to minimize the risk of misdiagnosis and delayed treatment.

\section{Case Presentation}

Received 02/15/2019

Review began 02/20/2019 Review ended 02/23/2019 Published 02/28/2019

\section{(c) Copyright 2019}

Vemulakonda et al. This is an open access article distributed under the terms of the Creative Commons Attribution License CC-BY 3.0., which permits unrestricted use, distribution, and reproduction in any medium, provided the original author and source are credited.
A 69-year-old male came with a complaint of cutaneous lesions on the right arm associated with redness and itching for 11 months. Other skin lesions and systemic symptoms were notably absent. A physical examination revealed three verrucous plaques on the right elbow and ulcerated papulonodules on the medial aspect of the right elbow (Figures 1-2). 


\section{Cureus}

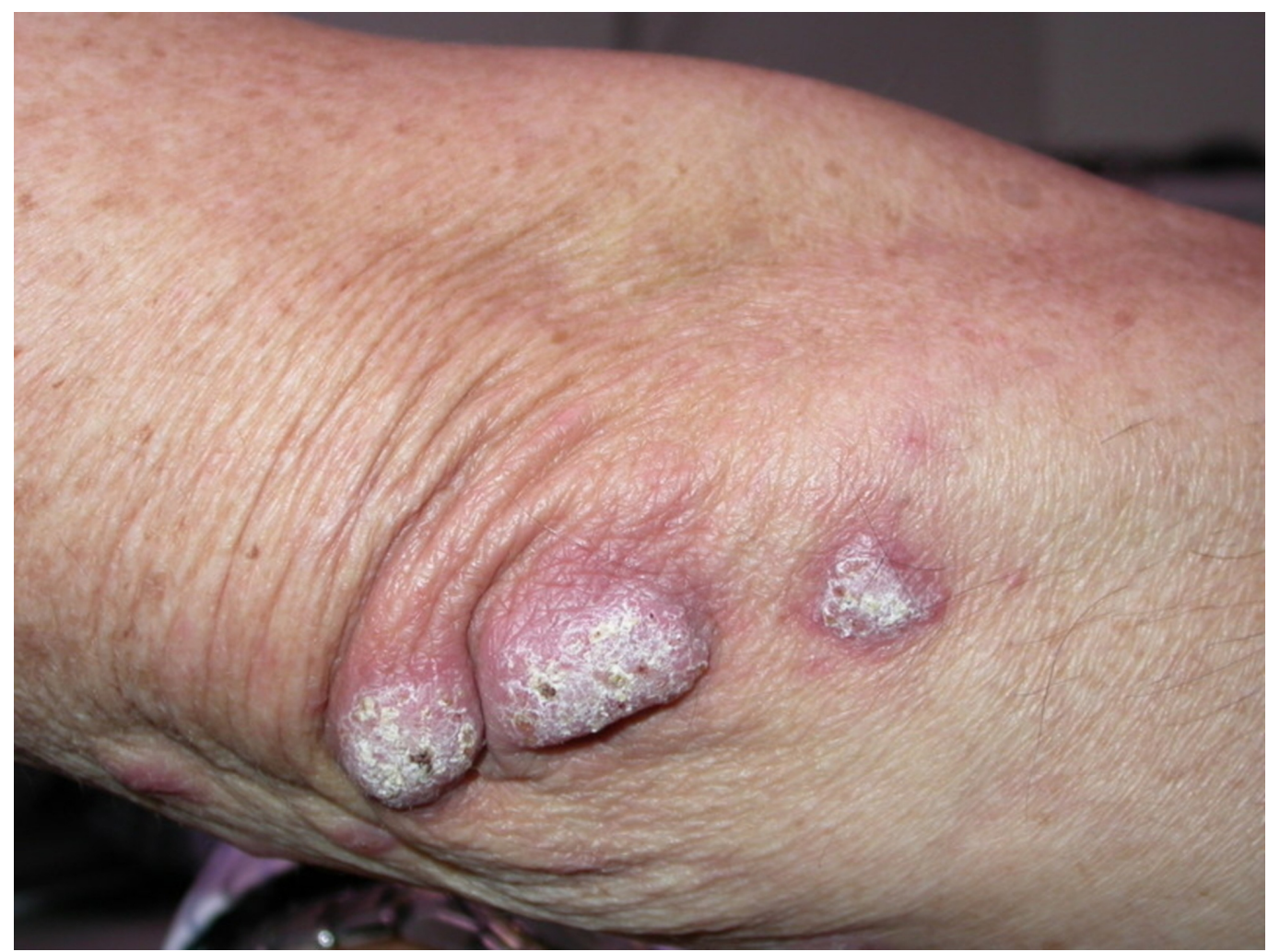

FIGURE 1: Three verrucous plaques on the right elbow of the patient

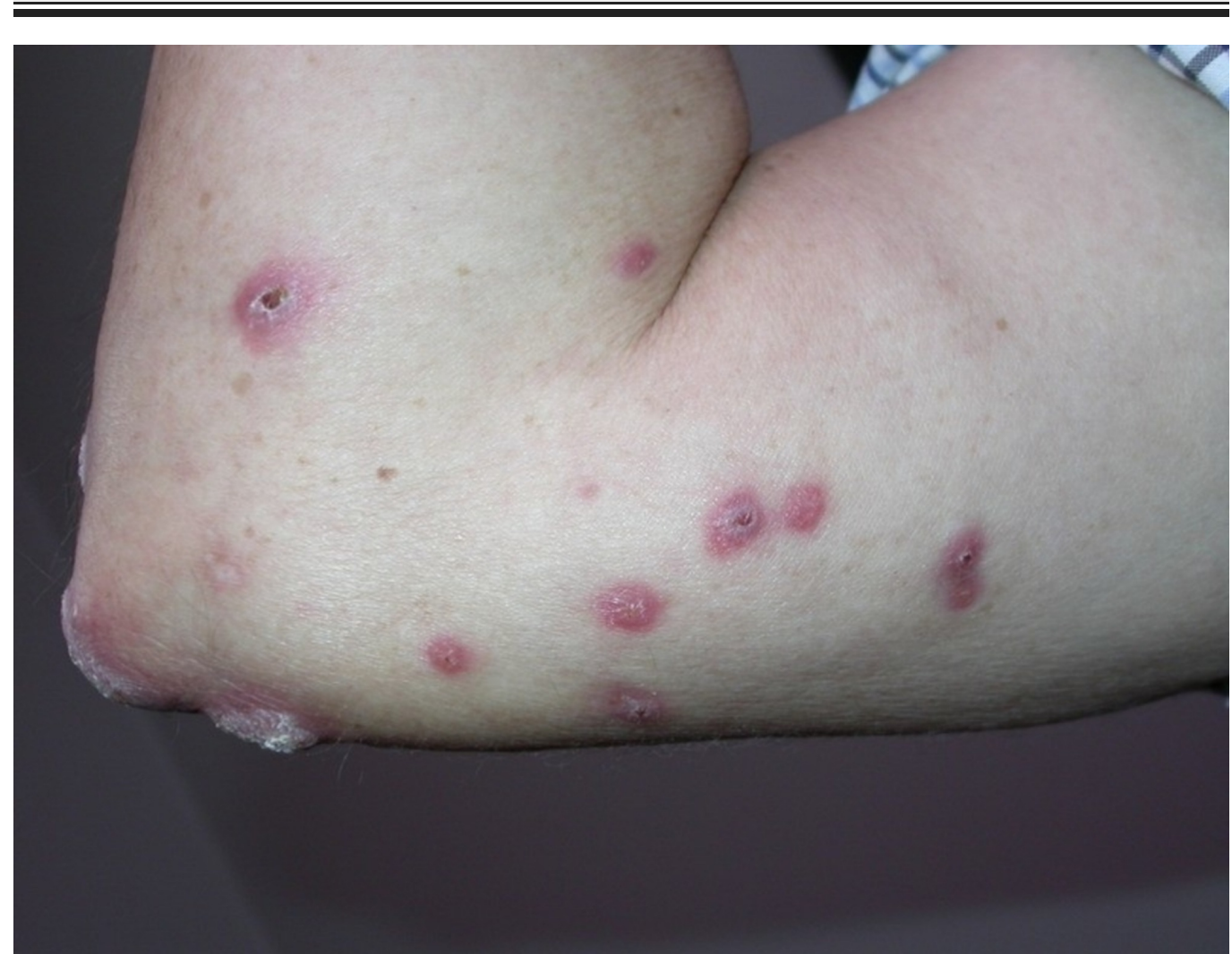

FIGURE 2: Ulcerated papulonodules on the medial aspect of the right elbow of the patient 


\section{Cureus}

A $4 \mathrm{~mm}$ punch biopsy from the medial aspect of the right elbow demonstrated chronic granulomatous inflammation with diffuse dermal mixed infiltrate of neutrophils, histiocytes, and plasma cells and occasional microabscesses (Figures 3-4). Gram stain, acid-fast bacilli (AFB), and periodic acid-Schiff (PAS) stains with appropriate controls for organisms were negative. Cultures obtained were negative for fungus and AFB at six weeks.

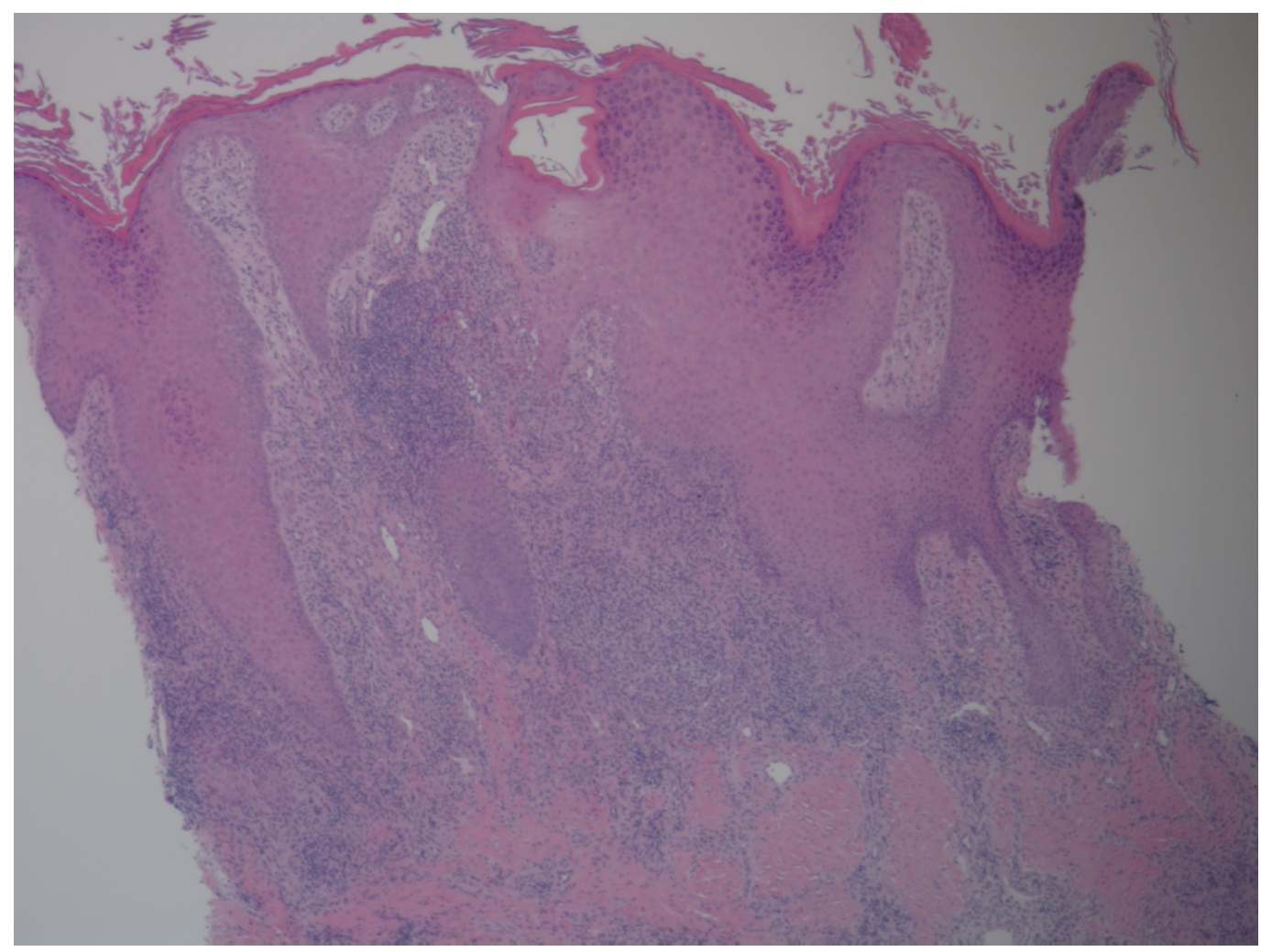

FIGURE 3: Histopathological examination in low power showing acanthosis, hyperkeratosis, and chronic granulomatous inflammation 


\section{Cureus}

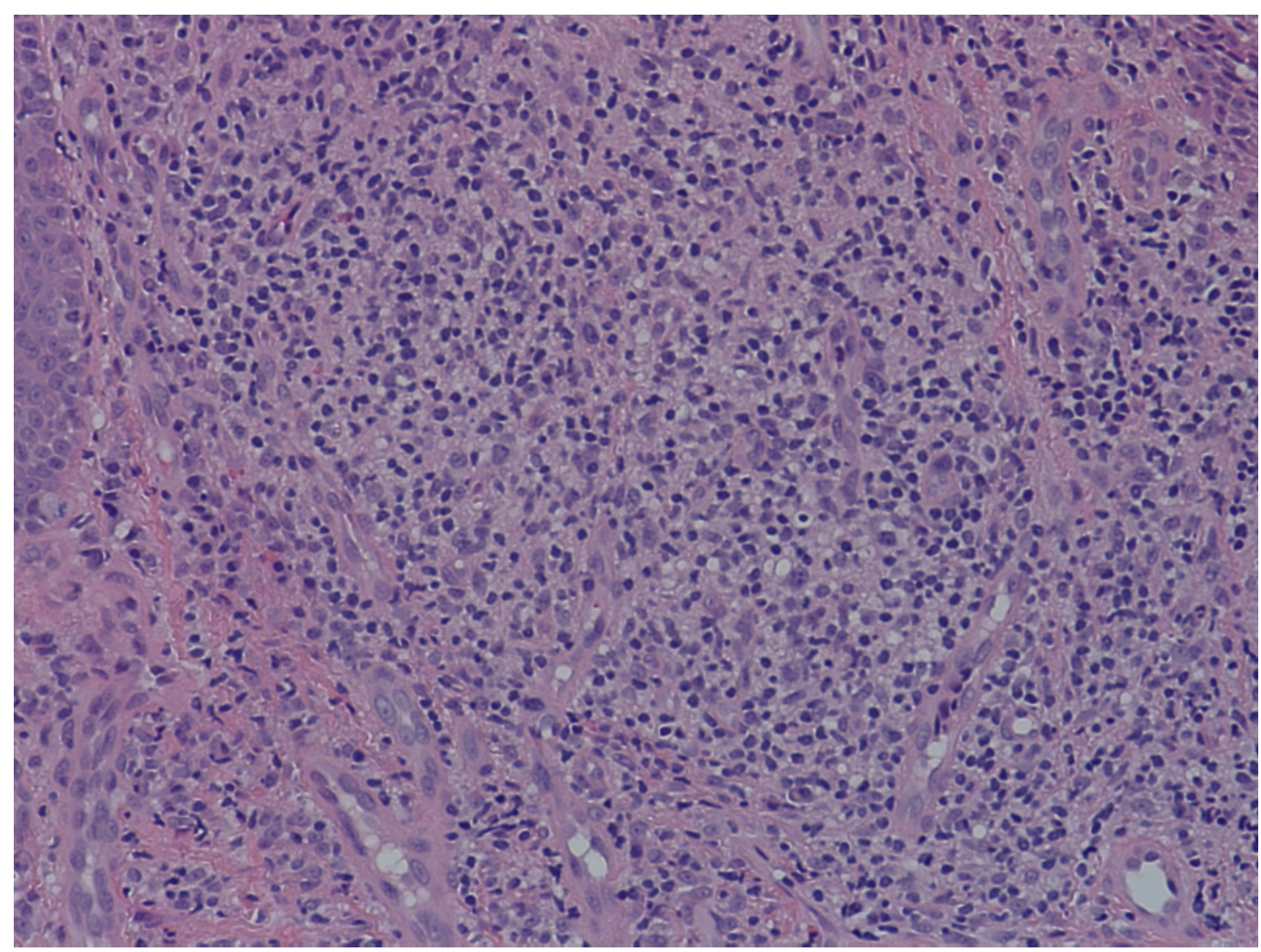

FIGURE 4: Histopathological examination in high power showing diffuse mixed inflammatory infiltrate in the dermis

A diagnosis of sporotrichosis was made based on the sporortrichoid spread of lesions in a linear pattern up the lymphatics, histopathological findings, and the absence of AFB on special stains and culture. The patient was started on itraconazole.

After a month, the patient came back for a follow-up with a flare-up of the lesions on the right elbow. On probing, the patient admitted to cleaning an aquarium at home before the start of lesions. A biopsy of the lesions was sent for AFB stain, PAS stain, and culture for fungus and AFB. The fungal stain showed no yeast and hyphae. No AFB were found on direct smear. No fungus was isolated at six weeks of culture. At seven weeks of culture, AFB was isolated and identified as Mycobacterium marinum (M. marinum). The patient was started on rifampin, 300 mg twice a day, and ethambutol, $400 \mathrm{mg}$ five times a day. The patient reported considerable improvement with the above treatment.

\section{Discussion}

M. marinum is a non-motile, non-spore forming, slow-growing photochromatic AFB. It produces a yellow pigment when exposed to light. $M$. marinum was reported as the most common cutaneous, nontuberculous mycobacterial infection with an incidence of 0.04 to 0.27 per 100,000 inhabitants [2]. The first human case of $M$. marinum isolated from skin lesions of swimmers who bathed in a contaminated pool in Sweden was reported by Norden and Linell in $1951[3]$.

M. marinum infection, also known as aquarium granuloma, swimming pool granuloma, or fish tank granuloma, can be contracted through minor abrasions incurred while bathing in swimming pools, ocean, or lake water or while cleaning home aquariums. A thorough history of 
the disease with a review of occupational and background exposure to potential pathogens during recent travels and leisure activities will help in the diagnosis of the infection.

M. marinum can clinically present as painful or painless, solitary or multiple erythematous papules, nodules, plaques, or verrucous lesions with or without crust or ulceration. In some instances, a sporotrichoid spread without accompanying lymphadenopathy has been reported [4]. The lesions can be categorized into three types to guide the treatment:

- Type 1 lesions: these are 1 - $2 \mathrm{~cm}$ superficial erythematous popular or verrucous self-limited lesions. Treatment with antibiotics may be required if remission is not observed.

- Type 2 lesions: these are subcutaneous granulomas that require treatment with antibiotics.

- Type 3 lesions: these are lesions that involve deeper structures, like tendons, bones, and joints, and require surgical debridement, along with antibiotic therapy [5].

Histopathological findings can range from a non-specific inflammatory infiltrate composed of neutrophils, monocytes, and macrophages to tuberculoid granulomas depending on the duration of the lesion. Hyperplastic or ulcerated epidermis with microabscesses can be found. Acid-fast stains may reveal organisms, but the sensitivity of acid-fast stains on biopsy material is low.

A culture of the involved tissue is crucial for establishing the diagnosis of M. marinum infection. The culture should be obtained even in the absence of microscopic evidence of bacilli, as the lesions have a very low concentration of microorganisms. The laboratory should be notified that $M$. marinum infection is suspected. Cultures are incubated in LowensteinJensen agar at $28^{\circ}$ to $32^{\circ} \mathrm{C}$ (in addition to $37^{\circ} \mathrm{C}$ ) and observed for six to 12 weeks [6]. The positivity rate of culture ranges from $70 \%$ to $80 \%$; hence, as illustrated in the presented case, organisms may not be recovered from the first culture performed and additional biopsy material for culture may be needed [7].

Recent advances in molecular methods allow definitive and rapid identification of M. marinum. Polymerase chain reaction (PCR) amplification techniques using mycobacterium genus-specific primers can be used to diagnose an M. marinum infection directly in the biopsy sample. Insolution hybridization or solid format reverse hybridization assays enable identification in the early stage of bacterial growth [8].

Management of the infection depends on the severity of the lesions. Early lesions are usually self-limited. They can resolve spontaneously or with antibiotic monotherapy, such as clarithromycin, azithromycin, moxifloxacin, tetracyclines, or trimethoprim/sulfamethoxazole. Severe infections with a sporotrichoid distribution or extensive infections with deeper structure involvement require multidrug therapy with rifampicin and ethambutol or clarithromycin in association with rifampicin or ethambutol. The duration of treatment may vary between six weeks to six months with treatment often continuing one to two months after the clinical remission. Surgical debridement is required in cases with subcutaneous tissue involvement [9].

\section{Conclusions}

In summary, the present report describes a case of an M. marinum infection in a 69-year-old immunocompetent male with cutaneous lesions on the right elbow for 12 months.

Histopathology detected chronic granulomatous inflammation with no evidence of fungi or AFB. The culture, in an appropriate medium, confirmed the diagnosis and treatment with rifampicin and ethambutol was effective. 
The diagnosis and management of an M. marinum infection are difficult without a proper history and a high index of suspicion for the disease. Patients presenting with indolent nodular skin infections affecting the upper extremities should be questioned about aquatic exposure. Tissue biopsy for histopathologic examination and culture in the appropriate medium, even in the absence of AFB in special stains, is essential to establish an early diagnosis and promptly initiate the appropriate therapy.

\section{Additional Information \\ Disclosures}

Human subjects: Consent was obtained by all participants in this study. NA issued approval NA. NA. Conflicts of interest: In compliance with the ICMJE uniform disclosure form, all authors declare the following: Payment/services info: All authors have declared that no financial support was received from any organization for the submitted work. Financial relationships: All authors have declared that they have no financial relationships at present or within the previous three years with any organizations that might have an interest in the submitted work. Other relationships: All authors have declared that there are no other relationships or activities that could appear to have influenced the submitted work.

\section{References}

1. Akram SM, Aboobacker S: Mycobacterium marinum. StatPearls. StatPearls Publishing, Treasure Island, FL; 2018. (Internet).

2. Sette CS, Wachholz PA, Masuda PY, da Costa Figueira RB, de Oliveira Mattar FR, Ura DG: Mycobacterium marinum infection: a case report . J Venom Anim Toxins Incl Trop Dis. 2015, $21: 7$.

3. Nordén A, Linell F: A new type of pathogenic Mycobacterium . Nature. 1951, 168:826. 10.1038/168826a0

4. Abudu B, Cohen PR: Sporotrichoid keratoacanthomas: case report and review of neoplasms presenting in a sporotrichoid pattern. Cureus. 2018, 10:3196. 10.7759/cureus.3196

5. Krooks J, Weatherall A, Markowitz S: Complete resolution of Mycobacterium marinum infection with clarithromycin and ethambutol: a case report and a review of the literature. J Clin Aesthet Dermatol. 2018, 11:48-51.

6. Dolenc-Voljc M, Zolnir-Dovc M: Delayed diagnosis of Mycobacterium marinum infection: a case report and review of the literature. Acta Dermatovenerol Alp Pannonica Adriat. 2010, 19:35-39.

7. Steinbrink J, Alexis M, Angulo-Thompson D, Ramesh M, Alangaden G, Miceli M: Mycobacterium marinum remains an unrecognized cause of indolent skin infections. Cutis. 2017, 100:331-36.

8. Piersimoni C, Scarparo C: Extrapulmonary infections associated with nontuberculous mycobacteria in immunocompetent persons. Emerg Infect Dis. 2009, 15:1352-58. 10.3201/eid1509.081259

9. Veronese F, Zavattaro E, Farinelli P, Colombo E, Savoia P: An unusual cutaneous infection caused by Mycobacterium marinum. JMM Case Rep. 2017, 4:e005088. 10.1099/jmmcr.0.005088 